\title{
Modified LEACH and Fuzzy C-Means Based Clustering Protocol for Wireless Sensor Networks
}

\author{
Tin Aye Chit, Nyein Aye Maung Maung, Khine Thin Zar \\ Department of Computer Engineering and Information Technology \\ Yangon Technological University \\ Yangon, Myanmar
}

\begin{abstract}
Wireless Sensor Networks formed by grouping of small batteries equipped sensor nodes, faces the major issue in optimization of energy when designing the routing protocols. Clustering based routing algorithm proved energy efficiency in Wireless Sensor Networks (WSNs), in which appropriate cluster formation and cluster heads (CHs) selection is crucial for energy saving. Thus, this paper proposes an approach which uses fuzzy C-Means (FCM) in cluster formation and uses the three parameters for cluster head selection: the new energy parameter considered threshold value, the residual energy of each node and the distance from node to base station (BS). FCM algorithm creates uniform clusters in the network area and reduces the distances between each node and their corresponding cluster head node. The proposed clustering protocol can give longer network lifetime when it is implemented in MATLAB simulation environment and compared to LEACH. Simulation results tell that the proposed protocol overcome the performance of LEACH protocol in terms of saving energy and reducing dead nodes to prolong the network lifetime.
\end{abstract}

Key Words: Wireless Sensor Networks, Fuzzy C-Means, LEACH, Cluster formation, Cluster head selection.

\section{INTRODUCTION}

Wireless Network is also a type of computer network in which a number of nodes are communicated without having a physical connectivity between these nodes. Wireless Sensor Network is a kind of Wireless Networks which have the same working principle as the conventional Wireless Networks. Because of technological advancement in wireless sensor networks (WSNs), it has been widely used as a standard information gathering tool in a wide range of applications, such as environmental monitoring, industrial applications, target tracking, battlefield surveillance, home security, precision agriculture, animal tracking and health monitoring. Sensor nodes with limited resources in terms of energy, memory, and computation are connected together to form a WSN. Each sensor node is employed with a small battery. This battery can store some amount of initial energy, and a fraction of it is consumed in every communication. During the network lifetime, many communications are taken place and the battery will be exhausted due to these communications. When WSNs are placed in harsh environment or in a kind of environments where it is difficult to reach, in most of the cases there is no way to recharge these batteries. This is the fact that minimizing energy consumption when designing the routing protocol is very important to increase the lifetime of WSNs.

Clustering protocols are the best solution for decreasing the energy use in WSNs. By using these protocols, the nodes in WSNs are divided into clusters and each node has their role such as cluster head $(\mathrm{CH})$ and cluster members. The cluster head node collects data from all other cluster members and forwards these data to the base station (BS). Cluster head $(\mathrm{CH})$ uses more energy than other member nodes in their cluster and the life of the network depends on that $\mathrm{CH}$. So, the duty of cluster head $(\mathrm{CH}) \mathrm{must}$ be assigned in rotational manner to share energy consumption and to design the protocols that can balance energy consumption.

This paper is composed of five sections. Section II presents related works on clustering protocols and Section III presents the detail of proposed work including the explanations of background theory. In section IV, simulation scenario and their results and analysis are shown. Finally, section V concludes this paper. 


\section{RELATED WORKS}

There are many protocols to extend the lifetime of WSNs and the most famous is Low Energy Adaptive Clustering Hierarchy Protocol (LEACH) that was proposed by Heinzelman et.al [1]. In LEACH, cluster head selection is based on randomized probability without considering the residual energy and proximity to the BS of that node. On the basis of LEACH protocol LEACH-C was proposed by Xinhua et.al [2], in which the formation of clusters is performed using centralized algorithm by the base station (BS). It solved the problem of LEACH by electing cluster head $(\mathrm{CH})$ on the basis of residual energy and distance from BS. The overall performance of LEACH-C is better than LEACH but it is sensitive to BS location. Ahlawat et.al [3] proposed a protocol, vice-CH LEACH (V-LEACH) to avoid base station overhead by considering the second $\mathrm{CH}$. It uses the minimum distance and maximum residual energy to extend the network lifetime and the vice- $\mathrm{CH}$ acts as $\mathrm{CH}$ when a main $\mathrm{CH}$ fails. V-LEACH gives satisfactory performance than LEACH and LEACH-C. However, VLEACH itself faces the extra overhead for choosing the vice- $\mathrm{CH}$ because it chooses vice- $\mathrm{CH}$ whenever it is necessary or not. A hybrid algorithm called LEACH-VF [7] proposed by Taqieddin et.al, uses two types of virtual forces: attractive and repulsive force. This algorithm reduces energy for communication by using attractive force and eliminates cluster overlapping by using repulsive force and thus it can maximize the coverage area. This algorithm still has the weakness of low energy efficiency and non-uniform distribution of energy among nodes. Khedri et.al [12] developed a new energy efficient algorithm called OLEACH. In this algorithm, a node is selected as cluster head if it has the energy greater than $10 \%$ of the initial energy but other influential parameters such as distance are not considered.

Bouyer et.al [13] created optimum number of CHs in LEACH algorithm by using Fuzzy C-Means (FCM) algorithm to reduce energy and to prolong the network lifetime. In this hybrid algorithm, each node compares its status based on three basic characteristic of energy, distribution and the center localization with the status of the other nodes in the neighbor nodes and if the energy of it is more than the neighbor node, introduces itself as the $\mathrm{CH}$ node and then the clustering starts by joining the nodes to the nearest cluster. Alami et.al [14] created the protocol named CAFL, in which the residual energy and closeness to base station value of each node are used to compute the chance value during $\mathrm{CHs}$ selection process. The sensor node which has the highest chance value will become $\mathrm{CH}$ for the current round. After selecting $\mathrm{CHs}$, they send advertisement message over sensors network to inform remaining sensor nodes that it is the $\mathrm{CH}$ and the cluster formation task is done. Therefore, energy consumption is decreased in network which means the lifetime of WSNs is maximized.

All research works have their idea and solutions for improving the network lifetime of WSNs. But there could have many other approaches for increasing WSNs' lifetime and the proposed hybrid protocol that combined Fuzzy C-Means and RED_LEACH is unique in its own method, in which cluster heads ( $\mathrm{CHs}$ ) are elected based on both node's residual energy and closeness to base station (BS). The detailed process of the proposed protocol is explained in further section.

\section{PROPOSED PROTOCOL}

The purpose of proposed protocol is to reduce the weakness of LEACH protocol and to create a new protocol that can prolong the life time of wireless sensor network. LEACH protocol, although it prolongs the network lifetime than static routing, still has some weakness. The LEACH protocol weakness are as follows. The random method is used to select the cluster heads, each sensor node in the network chooses a random number between 0 and 1 . If the chosen number of that node is less than the threshold value $\mathrm{T}$ (n), the node becomes a $\mathrm{CH}$ for the current round. T (n) can be computed using "(1)". So, the optimal number of cluster heads and distribution of them cannot be made sure. There has the equal priority to become the cluster heads (CHs) between the nodes which have low remaining energy and the node which have high remaining energy. If the nodes with low residual energy is chosen as the cluster heads then these nodes will die first. This weakness causes in shortening the life of sensor network.

$$
T(n)=\left\{\begin{array}{l}
\frac{P}{1-\mathrm{P} *\left(\mathrm{r} \times \bmod \left(\frac{1}{\mathrm{P}}\right)\right)}, \text { ifn } \in \mathrm{G} \\
0, \text { otherwise }
\end{array}\right.
$$

In the above equation, $r$ stands for current round number, $\mathrm{P}$ stands for desired percentage of cluster head $(\mathrm{CH})$ in this round, and $\mathrm{n}$ stands for the number of sensor nodes in the network. G stands for the set of nodes which does not become cluster head $(\mathrm{CH})$ in this current round.

After selecting the cluster heads and forming the cluster for the network, the data is transmitted from the cluster member nodes to their corresponding cluster head $(\mathrm{CH})$ node. The cluster member nodes in each cluster connect with heir cluster head node through a single hop transmission method. Each cluster head $(\mathrm{CH})$ in the network gathers all the data from its members and sends on them to the base station (BS). After a single round which starts from the cluster heads selection and ends by sending the sensed data to the base station is done, the network goes rounds by rounds until the last node die.

To refrain from the weakness of LEACH protocol and to improve the distribution of cluster heads node, the proposed protocol uses the fuzzy clustering method called Fuzzy C-Means clustering. The soft clustering fuzzy C-means (FCM) algorithm is firstly 
emerged by Dunn [15] in 1973 and upgraded by Bezdek et.al [16] in 1981. FCM is also an unsupervised clustering algorithm and has the same objective of cluster partitioning as the traditional k-means algorithm. This algorithm assigns the membership value to each sensor node based on their remoteness to the cluster center. The node which is situated close to the cluster center has the greater membership value than the node which is situated far away from the cluster center. The aim of FCM for cluster formation is to minimize the following objective function:

$$
\mathrm{J}_{\mathrm{m}}=\sum_{\mathrm{i}=1}^{\mathrm{N}} \sum_{\mathrm{j}=1}^{\mathrm{c}} \mathrm{u}_{\mathrm{ij}}^{\mathrm{m}}\left\|\mathrm{x}_{\mathrm{i}}-\mathrm{c}_{\mathrm{j}}\right\|^{2}
$$

where $\mathrm{c}$ represents the number of clusters, $\mathrm{N}$ represents the number of sensor nodes. The value of $\mathrm{m}$ must be greater than 1 , $\mathrm{u}_{\mathrm{ij}}$ is the membership degree of the sensor node $x_{i}$ belonging to cluster $j$, and $c_{j}$ is the center of the jth cluster. Fuzzy C-Means clustering is carried out by updating the above objective function in iterative manner. The membership $\mathrm{u}_{\mathrm{ij}}$ and the cluster centers $\mathrm{c}_{\mathrm{j}}$ is updated by the following equation:

$$
\begin{gathered}
\mathrm{u}_{\mathrm{ij}}=\frac{1}{\sum_{\mathrm{k}=1}^{\mathrm{c}}\left(\frac{\left\|\mathrm{x}_{\mathrm{i}}-\mathrm{c}_{\mathrm{j}}\right\|}{\left\|\mathrm{x}_{\mathrm{i}}-\mathrm{c}_{\mathrm{k}}\right\|}\right)^{\frac{2}{\mathrm{~m}-1}}} \\
\mathrm{c}_{\mathrm{j}}=\frac{\sum_{\mathrm{i}=1}^{\mathrm{N}} \mathrm{u}_{\mathrm{ij}}^{\mathrm{m}} \cdot \mathrm{x}_{\mathrm{i}}}{\sum_{\mathrm{i}=1}^{\mathrm{N}} \mathrm{u}_{\mathrm{ij}}^{\mathrm{m}}}
\end{gathered}
$$

The cluster formation by FCM guarantee the clustering to be perfect. After using the FCM algorithm for cluster formation stage, the proposed approach links to cluster head selection stage. The proposed protocol utilizes the residual energy of each node and the distance between each node and the base station in selecting the cluster heads, to reduce the drawbacks of LEACH: unbalanced spreading of cluster head nodes in the network area and probability of selecting a low residual energy node to become a $\mathrm{CH}$. In the proposed protocol, a node has the random value between 0 and 1, that number must be less than the threshold value calculated by (5) and it has the distance to base station (BS) value that is less than the mean value of all distance will become the cluster head $(\mathrm{CH})$.

$$
\mathrm{T}(\mathrm{n})=\left\{\begin{array}{l}
\frac{\mathrm{P}}{1-\mathrm{P} \times\left(\mathrm{r} \times \bmod \left(\frac{1}{\mathrm{P}}\right)\right)} \times \mathrm{E}_{\mathrm{r}}(\mathrm{i}), \text { ifn } \in \mathrm{G} \\
0, \text { otherwise }
\end{array}\right.
$$

The above equation is based on the threshold equation of LEACH protocol and $E_{r}(i)$ value is included. $E_{r}(i)$ stands for the residual energy of each node and it is used to multiply with the original threshold value to reduce the dead nodes in order to extend the network lifetime. The consumed energy or dissipated energy of each node is subtracted from the original energy value of the corresponding node to get the residual energy of each node. The dissipated energy for sending m-bits message is calculated using the following equation:

$$
\mathrm{E}_{\mathrm{TX}}(\mathrm{m}, \mathrm{d})=\left\{\begin{array}{l}
\mathrm{m} \times \mathrm{E}_{\mathrm{elec}} \times \mathrm{m} \times \varepsilon_{\mathrm{fs}} \times \mathrm{d}^{2}, \text { ifd } \leq \mathrm{d}_{0} \\
\mathrm{~m} \times \mathrm{E}_{\mathrm{elec}} \times \mathrm{m} \times \varepsilon_{\mathrm{mp}} \times \mathrm{d}^{4}, \mathrm{ifd}>\mathrm{d}_{0}
\end{array}\right.
$$

where $\mathrm{E}_{\mathrm{TX}}(\mathrm{m}, \mathrm{d})$ stands for the energy consumed for transmitting $\mathrm{m}$-bits message with a distance $\mathrm{d}$, and $\mathrm{E}_{\text {elec }}$ is the transceiver circuit running energy for one bit. The amplifier parameter $\varepsilon_{\mathrm{fs}}$ is for the free space propagation model and the amplifier parameters $\varepsilon_{\mathrm{mp}}$ is for the two-ray ground propagation model. The two-way ground model is utilized when the distance between the transmitter and the receiver is larger than the threshold distance $\mathrm{d} 0$. If the distance is smaller than or equal to the distance $\mathrm{d} 0$, the free space model will be used. The threshold distance d0 between two-ray ground propagation model and free space propagation model can be obtained from:

$$
\mathrm{d}_{0}=\sqrt{\frac{\varepsilon_{\mathrm{fs}}}{\varepsilon_{\mathrm{mp}}}}
$$

The receiver circuit running energy for m-bits message is:

$$
\mathrm{E}_{\mathrm{RX}}(\mathrm{m})=\mathrm{m} \times \mathrm{E}_{\mathrm{elec}}
$$

If a node matches the following facts, it will become the cluster head $(\mathrm{CH})$ for the current round: the greater residual energy than the mean residual energy, the smaller random value than the threshold value calculated by (5) and the shorter distance between that node and base station than the mean distance. The $\mathrm{CH}$ broadcasts advertisement message to all nodes and the network indicating that it has $\mathrm{CH}$ node and invites other nodes to join it and forms a cluster. 
International Journal of Advances in Scientific Research and Engineering (ijasre), Vol 7 (1), January -2021

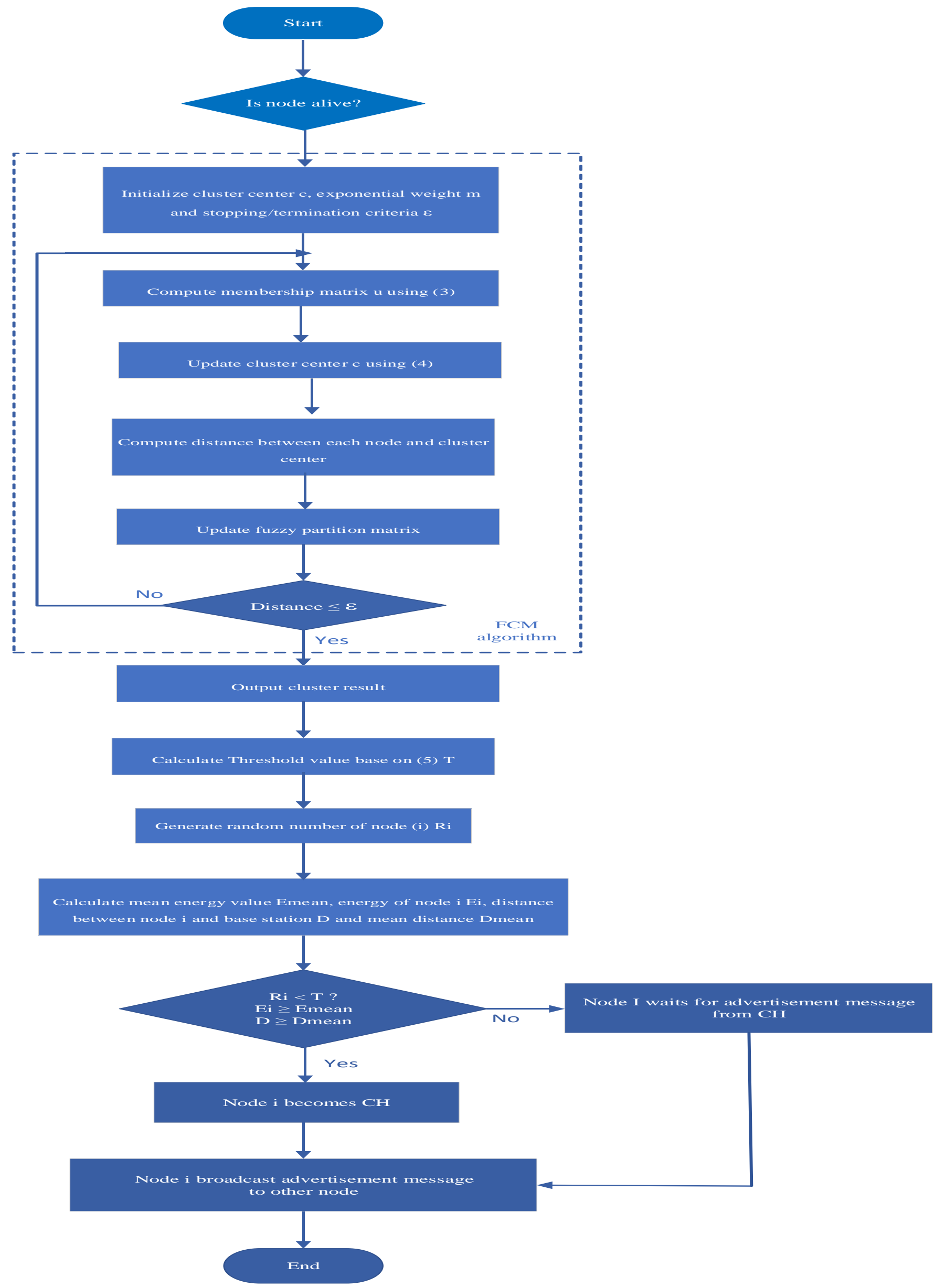

Figure1: System flow diagram of proposed protocol 


\section{SIMULATION AND RESULTS ANALYSATION}

MATLAB software is used for the development of proposed protocol and simulations using the network model which consists of 100 sensor nodes deployed over a field of $(100 \mathrm{M} \times 100 \mathrm{M})$ square unit network area. The detail scenario for simulation is presented in Table 1.1. The proposed protocol is compared with the original LEACH protocol using the two performance-matrix: sum of energy of nodes vs rounds and number of dead-nodes vs rounds.

Table1.1. Scenario for simulation

\begin{tabular}{|l|l|}
\hline \multicolumn{1}{|c|}{ Parameters } & \multicolumn{1}{c|}{ Value } \\
\hline Initial Energy E0 & 0.5 Joules \\
\hline Electronic Energy Eelec & $50 \mathrm{~nJ} / \mathrm{bit}$ \\
\hline Free Space Model Efs & $10 \mathrm{pJ} / \mathrm{bit} / \mathrm{m} 2$ \\
\hline Multipath Model Emp & $0.0013 \mathrm{pJ} / \mathrm{bit} / \mathrm{m} 4$ \\
\hline Data Aggregation Energy EDA & $5 \mathrm{~nJ} / \mathrm{bit} / \mathrm{signal}$ \\
\hline No. of node & 100 \\
\hline No. of round & 500,1000 \\
\hline
\end{tabular}

The sensing network area is covered by the 100 sensor nodes in random fashion. The 100 sensor nodes are clustered by Fuzzy CMeans (FCM) and the cluster head ( $\mathrm{CH})$ for each cluster is chosen by using the new threshold value, the node's residual energy value that is greater than the average energy value and the node's distance to the base station (BS) that is less than the mean distance. Figure 2 shows the clustered wireless sensor network by proposed protocol, in which the black spots represent the cluster center, the colored circles represent the cluster member nodes and the red crosses represent the cluster heads. The green circle represents the base station which is positioned at the center of the network area. In this figure, the seven clusters are created for the sensor nodes to distribute in the network area by using the FCM algorithm. It can be seen that the symmetric clusters are deployed in the sensing area.

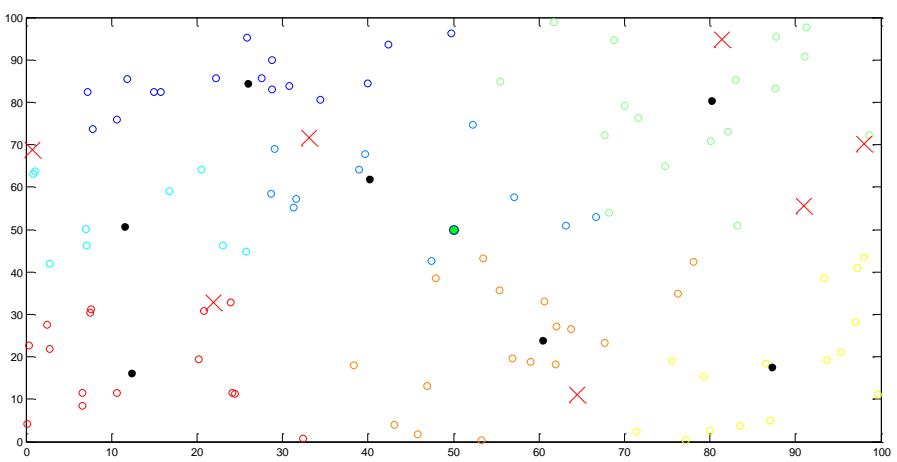

Figure2: Cluster creation by proposed protocol

Firstly, the proposed protocol and LEACH protocol are compared using the sum of energy of node value. As illustrated in figure 3, the proposed protocol does not reduce energy till 500 rounds but LEACH protocol reduces energy reaching the sum of energy to 30 Joules after 500 rounds. The proposed protocol still efficient till 1000 rounds by seeing the figure 4, in which figure the blue colored line for the proposed protocol does not drop from 50 Joules but the green colored line for LEACH protocol sharply drops from 50 Joules to 10 Joules after 1000 rounds.

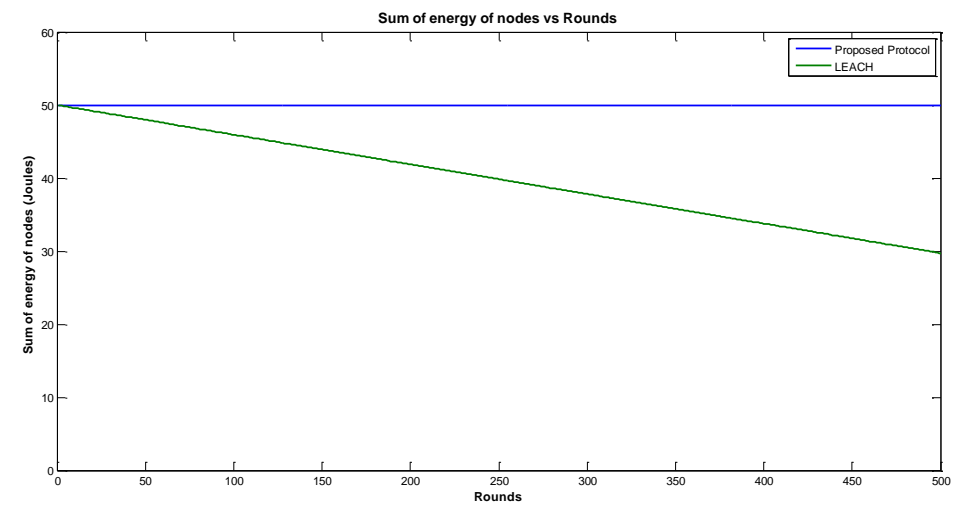

Figure3: Sum of energy of nodes in 500 rounds 


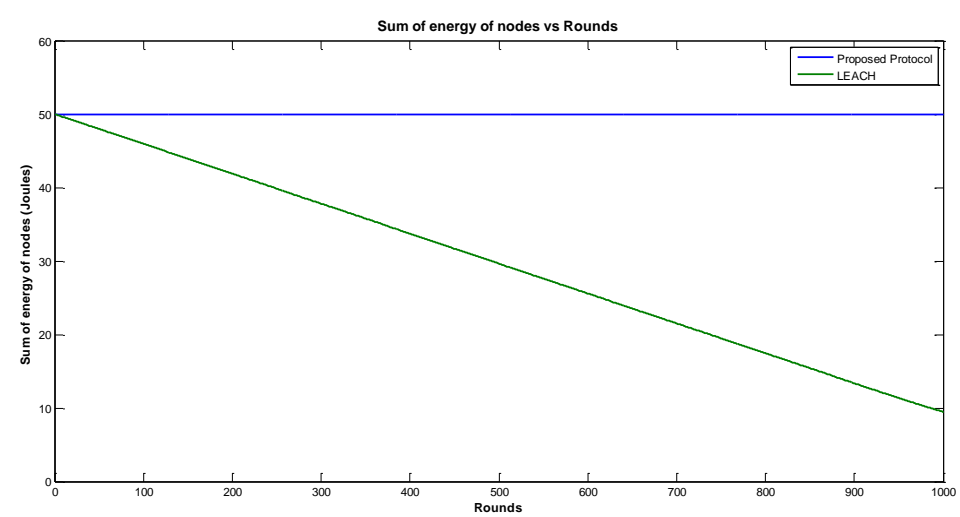

Figure4: Sum of energy of nodes in 1000 rounds

The illustrated results of figure 5 shows the number of dead nodes based on the number of rounds. The red line shows the results of LEACH protocol and the blue line shows the results of proposed protocol for the number of dead nodes in each round. The two protocols maintain without dying any node till 500 rounds but the first node died after 925 rounds by using LEACH protocol. The number of dead nodes increases round by round and 10 nodes died when 1000 rounds is reached by using LEACH protocol. The proposed protocol can maintain 100 nodes without dying till 1000 rounds.

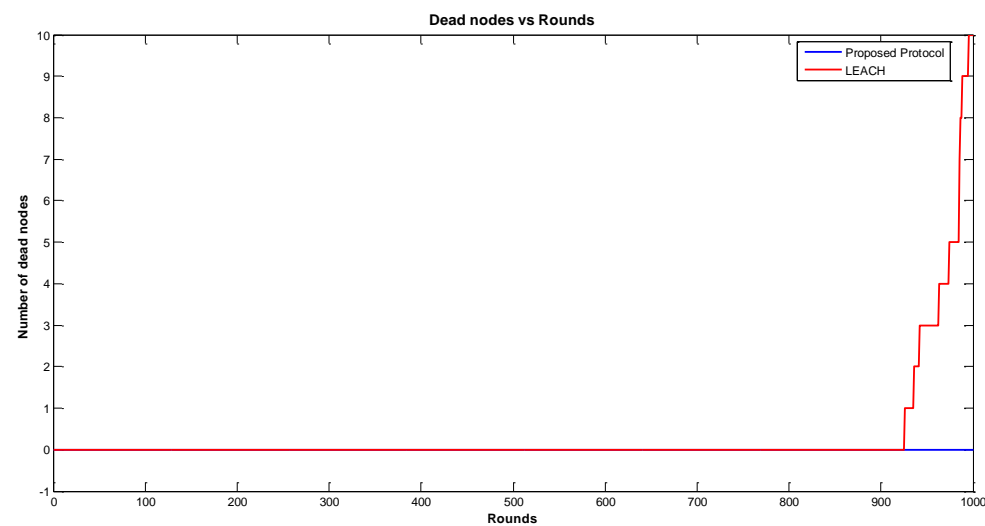

Figure5: Number of dead nodes in 1000 rounds

\section{CONCLUSIONS}

Optimization of energy consumption to extend the lifespan of wireless sensor network is the main objective of the research and because of this objective the energy efficient Fuzzy C-Means and LEACH based proposed protocol is designed and presented in this paper. In the proposed protocol, FCM is used for cluster formation to create uniform clusters in reducing the intra-cluster distance and the three parameters is used for cluster heads $(\mathrm{CHs})$ selection. Creating symmetric clusters causes the reducing in energy consumption and saves more energy than asymmetric cluster which results in extending the lifetime of WSNs. The simulation results proof that the proposed protocol performs better when it is compared with LEACH protocol in terms of sum of energy of nodes and number of dead nodes. In future, the proposed protocol will be improved for dynamic sensor nodes and it should be developed to use in real world applications.

\section{REFERENCES}

[1] Heinzelman, W.R., Chandrakasan, A. and Balakrishnan, H., 2000, January. Energy-efficient communication protocol for wireless microsensor networks. In Proceedings of the 33rd annual Hawaii international conference on system sciences (pp. 10-pp). IEEE.

[2] Xinhua, W. and Sheng, W., 2010, August. Performance comparison of LEACH and LEACH-C protocols by NS2. In 2010 Ninth International Symposium on Distributed Computing and Applications to Business, Engineering and Science (pp. 254258). IEEE.

[3] Ahlawat, A. and Malik, V., 2013, April. An extended vice-cluster selection approach to improve v leach protocol in WSN. In 2013 Third International Conference on Advanced Computing and Communication Technologies (ACCT) (pp. 236-240). IEEE.

[4] Javaid, N., Waseem, M., Khan, Z.A., Qasim, U., Latif, K. and Javaid, A., 2013, April. ACH: Away cluster heads scheme for energy efficient clustering protocols in WSNs. In 2013 Saudi International Electronics, Communications and Photonics Conference (pp. 1-4). IEEE. 
[5] Hou, R., Ren, W. and Zhang, Y., 2009, October. A wireless sensor network clustering algorithm based on energy and distance. In 2009 Second International Workshop on Computer Science and Engineering (Vol. 1, pp. 439-442). IEEE.

[6] Ding, X., Xie, F. and Wu, Q., 2009, August. Energy-balanced clustering with master/slave method for wireless sensor networks. In 2009 9th International Conference on Electronic Measurement \& Instruments (pp. 3-20). IEEE.

[7] Taqieddin, E., Awad, F. and Ahmad, H., 2017. Location-Aware and Mobility-Based Performance Optimization for Wireless Sensor Networks. J. Wirel. Mob. Networks Ubiquitous Comput. Dependable Appl., 8(4), pp.37-59.

Nam, D.H. and Min, H.K., 2007, August. An energy-efficient clustering using a round-robin method in a wireless sensor network. In 5th ACIS International Conference on Software Engineering Research, Management \& Applications (SERA 2007) (pp. 54-60). IEEE.

[8] Song, X., Wang, C., Wang, J. and Zhang, B., 2010, June. A hierarchical routing protocol based on AFSO algorithm for WSN. In 2010 International Conference on Computer Design and Applications (Vol. 2, pp. V2-635). IEEE.

[9] Peng, Z. and Li, X., 2010, July. The improvement and simulation of LEACH protocol for WSNs. In 2010 IEEE International Conference on Software Engineering and Service Sciences (pp. 500-503). IEEE.

[10] Muruganathan, S.D., Ma, D.C., Bhasin, R.I. and Fapojuwo, A.O., 2005. A centralized energy-efficient routing protocol for wireless sensor networks. IEEE Communications Magazine, 43(3), pp. S8-13.

[11] Bian, X., Liu, X. and Cho, H., 2008, August. Study on a cluster-chain routing protocol in wireless sensor networks. In 2008 Third International Conference on Communications and Networking in China (pp. 964-968). IEEE.

[12] Khediri, S.E., Nasri, N., Wei, A. and Kachouri, A., 2014. A new approach for clustering in wireless sensors networks based on LEACH. Procedia Computer Science, 32, pp.1180-1185.

[13] Bouyer, A., Hatamlou, A. and Masdari, M., 2015. A new approach for decreasing energy in wireless sensor networks with hybrid LEACH protocol and fuzzy C-means algorithm. International Journal of Communication Networks and Distributed Systems, 14(4), pp.400-412.

[14] El Alami, H. and Najid, A., 2020. Fuzzy logic-based clustering algorithm for wireless sensor networks. In Sensor Technology: Concepts, Methodologies, Tools, and Applications (pp. 351-371). IGI Global.

[15] Dunn, J.C., 1973. A fuzzy relative of the ISODATA process and its use in detecting compact well-separated clusters.

[16] Bezdek, J.C., Ehrlich, R. and Full, W., 1984. FCM: The fuzzy c-means clustering algorithm. Computers \& Geosciences, 10(2-3), pp.191-203. 